\title{
BMJ Open Patient, physiotherapist and surgeon endorsement of the core domain set for total hip and total knee replacement in Germany: a study protocol for an OMERACT initiative
}

To cite: Prill R, Singh JA, Seeber GH, et al. Patient, physiotherapist and surgeon endorsement of the core domain set for total hip and total knee replacement in Germany: a study protocol for an OMERACT initiative. BMJ Open 2020;10:e035207. doi:10.1136/ bmjopen-2019-035207

- Prepublication history for this paper is available online. To view these files, please visit the journal online (http://dx.doi. org/10.1136/bmjopen-2019035207).

Received 22 October 2019 Revised 26 February 2020 Accepted 26 May 2020
Check for updates

(C) Author(s) (or their employer(s)) 2020. Re-use permitted under CC BY-NC. No commercial re-use. See rights and permissions. Published by BMJ.

For numbered affiliations see end of article.

Correspondence to

Dr Robert Prill;

robert.prill@outlook.de

\section{ABSTRACT}

Introduction There is a lack of harmonising measures for clinical trials on total joint replacement (TJR) that would allow for results from TJR studies to be compared or pooled. The Outcome Measures in Rheumatology (OMERACT) TJR core domain set is already endorsed among patients and physicians in the USA and Australia. Physiotherapists use different types of measurements compared to orthopaedic surgeons while both make substantial contributions to research in the field of TJR. To achieve consensus on core measurements sets, patients, physiotherapists and orthopaedic surgeons need to achieve consensus on the core domains for TJR trials. Methods and analysis For this multistage study, first, the OMERACT TJR core domain set survey will be translated to German and validated according to WHO guidelines. Next, the TJR core domain set will be considered for endorsement in different German stakeholder groups including patients, physiotherapists and orthopaedic surgeons.

Ethics and dissemination Ethical approval for this protocol was given by the ethics committee of the Brandenburg University of Technology CottbusSenftenberg (BTU-CS, EK 2019-2). This article is based on the protocol version 2.5 from 6 May 2020. Anonymous data will be presented only. We will publish the results in peer-reviewed publications and at international conferences.

Trial registration number German Clinical Trials Registry (DRKS00016015).

\section{INTRODUCTION}

Osteoarthritis (OA) is a significant cause for disability. ${ }^{1}$ Total joint replacement (TJR) is a frequently used and evidently effective treatment for end stage OA, when other treatments have failed..$^{2-4}$ The knee and hip are the most commonly replaced joints. In 2016, over 135000 total hip replacements (THAs) and 105000 total knee replacements (TKAs) were performed in Germany. ${ }^{5}$ The
Strengths and limitations of this study

- This is the first study that cross-culturally validates the Outcome Measures in Rheumatology total joint replacement core domain set, thus establishing a German version for further clinical and research use.

- A German translation will provide broader insights outside English speaking countries.

- The iterative international process for core domain set development will help to produce generalisable data for stakeholder priorities.

- Germany only partly represents the cultural diversity of Europe.

American Joint Replacement registry reports more than 300000 THAs and more than half a million of TKAs for the same period in the USA. ${ }^{6}$ About 50000 THAs and 60000 TKAs were implanted that year in Australia. ${ }^{7}$ The Outcome Measures in Rheumatology (OMERACT) TJR working group, an international group of methodologists, patient partners, physiotherapists, orthopaedic surgeons and rheumatologists was established in $2008 .{ }^{8}$ This group performed systematic reviews, identifying a lack of comparability between TJR randomised trials. ${ }^{9}$ When combining the results of systematic reviews on this topic ${ }^{9-11}$ and the result of surveys and Delphi panels in different stakeholder groups (patients, surgeons, physical therapists and rheumatologists), the group derived a preliminary core domain set for clinical TJR trials for knee and hip OA at OMERACT 2014. ${ }^{12}$ This core domain set was developed using an iterative process over several years to ensure that important stakeholder priorities regarding core domains were not missed. ${ }^{13-17}$ While it is possible that new domains may be identified 
Table 1 Modified Standard Protocol Items: Recommendations for Interventional Trials figure of study schedule and design

\section{Study period}

\begin{tabular}{|c|c|c|c|c|c|c|}
\hline \multirow[b]{2}{*}{ Timepoint } & \multicolumn{4}{|c|}{$\begin{array}{l}\text { Step 1: validated questionnaire } \\
\text { translation }\end{array}$} & \multicolumn{2}{|c|}{$\begin{array}{l}\text { Step 2: endorsement } \\
\text { patients, physiotherapists } \\
\text { and orthopaedic surgeons } \\
\text { for outcome measures in } \\
\text { TJR }\end{array}$} \\
\hline & T1 & T2 & T3 & T4 & T1 & T2 \\
\hline \multicolumn{7}{|l|}{ Validated questionnaire translation } \\
\hline Backward translation & & & $x$ & & & \\
\hline Panel discussion with regard to differences to initial version & & & & $\mathrm{x}$ & & \\
\hline
\end{tabular}

Endorsement of core measurement domains in stakeholder groups

$\begin{array}{ll}\text { Patients } & X \\ \text { Physiotherapists } & X \\ \text { Orthopaedic surgeons } & X\end{array}$

Evaluation of consensus across stakeholder groups

Likert scale ratings of proposed core domains in stakeholder groups

TJR, total joint replacement.

during this study, they will have to achieve a threshold high enough to merit inclusion in the core domain set based on frequency (arbitrarily at $30 \%$ of the sample) and validation in another independent sample, preferably in another language.

OMERACT uses an onion model to signify the importance of areas that should be reported in clinical trials, where outcome domains placed in the inner circle are termed 'core' and are considered the most important ones to report. Consensus was reached on the inner critical circle, including pain, function, patient satisfaction, revision, adverse effects and death as the core domain set. Cost and participation are in the middle circle, and the range of motion is in the outer circle or research agenda. The next step now is to develop a core measurement set for TJR trials but a weakness in broad international acceptance of the core domains is the limited validation of the findings in Europe. Moreover, both orthopaedic surgeons and physiotherapist substantially contribute to research in the field of TJR. However, physiotherapists use different types of measurement instruments compared with orthopaedic surgeons. ${ }^{18}$ To harmonise TJR clinical trial outcomes, it is important that all stakeholders agree on the core domain areas and on the instruments used to measure these areas. However, no information about such agreement is yet available in Germany. Therefore, it is necessary to assess whether physiotherapists, patients and orthopaedic surgeons in Germany agree on the TJR core domain set.

\section{Methods and analysis}

The aim of the study is to achieve further consensus on the OMERACT TJR core domain set including three important stakeholder groups: patients, physiotherapists and orthopaedic surgeons in Germany. A multistage study is necessary to achieve this goal. In accordance to the OMERACT handbook, partners from at least three continents must be chosen in a first step. Studies previously have included patients from the USA and Australia. At the OMERACT meeting 2018 in Terrigal (Australia), the TJR group met and agreed to do another survey and validation in Germany, as an initial approach of representing European populations. The validated and successfully used OMERACT TJR core domain set survey will be used for an endorsement in these stakeholder groups.

Before the core domain survey can be used for data collection in a German speaking population, the original English version must first be translated to German and subsequently be validated in accordance to recognised

Table 2 Baseline characteristics of the three endorsement groups

$\begin{array}{lll}\begin{array}{l}\text { Orthopaedic } \\ \text { surgeons }\end{array} & \text { Physiotherapists } & \text { Patients } \\ \text { (N) } & \text { (N) } & \text { (N) }\end{array}$

Female, $\mathrm{n}(\%)$

Age, years, mean

(SD)

Age category, $\mathrm{n}(\%)$
$<44$
$45-54$
$55-64$
$65-74$
$\geq 75$

Data are presented as mean (SD) or $\mathrm{n}(\%)$ unless otherwise stated. 
Translation of the English

Version of OMERACT TJR

draft core domain set survey

Recruitment for each stakeholder group

\begin{tabular}{|c|c|}
\hline & $\begin{array}{c}\text { Physiotherapists } \\
n=10\end{array}$ \\
& $\begin{array}{c}\text { Validation of the German } \\
\text { Version of OMERACT TJR } \\
\text { draft core domain set } \\
\text { survey }\end{array}$ \\
\hline
\end{tabular}

Orthopaedic Surgeons

$\mathrm{n}=10$

\section{Recruitment for each stakeholder group}
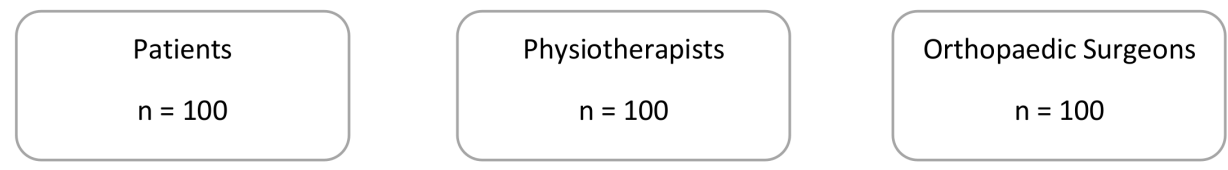

Endorsement

Consensus

Figure 1 Flow chart. OMERACT, Outcome Measures in Rheumatology; TJR, total joint replacement.

standards. The planed steps of the study are visualised in table 1 .

\section{Step 1: validation of the German version of the OMERACT TJR draft} core domain set survey

In accordance to the WHO recommendation for the process of translation and adaptation of instruments, ${ }^{19}$ a German version of the original English core domain survey will be established as described below. First two native German-speaking individuals, one medical professional and one lay person, will translate the original English version into German. Subsequently, a bilingual expert panel of the author's team will discuss the translated German versions and provide suggestions for improvement. Following this, two native English-speaking persons, one medical professional and one lay person, unaware of the original English version, will do a backtranslation. Any differences between the original English version and the forward-backward translated version will be discussed in the expert panel and the German wording will be adjusted if necessary. As soon as the bilingual expert panel regards the new German version acceptable, it will be pretested in five representatives from each stakeholder group (ie, patients, orthopaedic surgeons, physiotherapists). While filling out the survey, they will be systematically debriefed to state with their own words, what they think each question is asking for. Furthermore, they will be asked to explain how they chose their answer. This will be repeated for each survey item. Respondents will be asked to give feedback about each word they did not understand or found unacceptable. When wording discrepancies are identified, the respondents will be asked during in-depth personal interviews which of the alternatives fit best to their usual language. A written report of the pretesting will be provided by the TJR working group and the authors being involved in this protocol. This process will result in the final German version of the OMERACT TJR core domain set survey 1.0 (OMERACT TJR CDS-G1.0). As recommended by the WHO, the initial forward version, a summary of recommendations from the expert panel, the back translation, 
Table 3 Stakeholder ratings of different core domains

Orthopaedic surgeons (N)
Physiotherapists

(N)
Patients

(N)
$P$ value

Joint pain

Function or functional ability (ability to function in society, work;

work productivity, employability; disability; work disability)

Patient satisfaction (satisfaction with the outcome, satisfaction

with the procedure)

Revision surgery (including reoperation)

Adverse events (total and specific)

Death

Additional domains for consideration for reporting in TJR clinical trials

Patient participation

Cost

Data are presented as median (IQR) unless otherwise stated.

TJR, total joint replacement.

a summary of difficulties occurring during pretesting, the proposed clarifications and the final version will be documented and published.

Step 2: a three-armed endorsement in patients, physiotherapists and orthopaedic surgeons for outcome measures in TJR within rheumatology (OMERACT)

The research group (1) prospectively registered the study in the German Clinical Trials Register prior to starting the validation of a German OMERACT TJR core domain set survey, (2) developed the study protocol and (3) proposed for ethical approval. In a next step leading societies for patients, physiotherapists and orthopaedic surgeons specialised in TJR, as well as health centres with a focus on TJR will be informed about the upcoming endorsement.

\section{Study population and participant selection}

The study population will comprise (a) group A: patients with elective THA or TKA, (b) group B: physiotherapists with experience of treating patients with TJR and (c) group C: orthopaedic surgeons with a focus on TJR. Patients will be recruited by contacting patients from the registry of hospitals with a focus on TJR, so called 'Endoprothetikzentren der Maximalversorgung' (EPZmax). Physiotherapists will be recruited in two stages. First, the physiotherapy departments of EPZmax will be contacted and second, if not enough suitable participants take part, the leading physiotherapy societies 'Physio Deutschland', 'IFK - Bundesverband selbständiger Physiotherapeuten' and 'Deutsche Gesellschaft für Physiotherapiewissenschaft' will be contacted for additional participants using a snowball sampling approach. Orthopaedic surgeons will be recruited the same way, first contacting the EPZmax and second, the leading German society for orthopaedic surgery 'Arbeitsgemeinschaft Endoprothetik' (AE) and the 'Deutsche Gesellschaft für Orthopädie und Unfallchirurgie'.

Inclusion criteria

To be involved in the patient group A, participants must have undergone TKA or THA due to end stage OA, refractory to non-surgical treatments, within the last 2 years. In order to have a share in the physiotherapist group B, participants must be physiotherapist with at least 3 years of working experience in either the management of patients with TJR or research involving patients with TJR. In the orthopaedic surgeons group C, participants will be included if they state at least 3 years of experience in THA or TKA surgery or research including patients with THA or TKA.

\section{Exclusion criteria}

Participants of group A, B and C will be excluded, if they:

1. Fail to fill out at least $80 \%$ of the survey.

2. Are not identifiable as members of one of the three groups.

Participants of group A will be excluded, if they:

1. Were provided a THA or TKA due to an accident or non-OA cause.

2. Currently have major complications after THA or TKA surgery.

Participants of group B and C will be excluded, if they:

1. Are unfamiliar with the management or research in patients with TJR.

2. Have less than 3 years of work experience.

\section{Recruitment of participants}

Every potential group A participant will be selected randomly from the hospital registries until the estimated number of patients agreeing to participate is reached. For the group B sample, we will contact all German EPZmax 
Table 4 Stakeholder consensus of different core domains on three different subclassifications of patients with TJR

Patients with THA Patients with TKA

Core domains to be reported in every TJR clinical trial

(N)

(N)

P value

Joint pain

Function or functional ability (ability to function in society, work; work productivity, employability; disability; work disability)

Patient satisfaction (satisfaction with the outcome, satisfaction with the procedure)

Revision surgery (including reoperation)

Adverse events (total and specific)

Death

Additional domains for consideration for reporting in TJR clinical trials

Patient participation

Cost

Core domains to be reported in every TJR clinical trial

Females

(N)

Males

(N)

Joint pain

Function or functional ability (ability to function in society, work; work productivity, employability; disability; work disability)

Patient satisfaction (satisfaction with the outcome, satisfaction with the procedure)

Revision surgery (including reoperation)

Adverse events (total and specific)

Death

Additional domains for consideration for reporting in TJR clinical trials

Patient participation

Cost

Core domains to be reported in every TJR clinical tria

Patients $<55$ years Patients $\geq 55$ years $P$ value

(N)

(N)

Joint pain

Function or functional ability (ability to function in society, work; work productivity, employability; disability; work disability)

Patient satisfaction (satisfaction with the outcome, satisfaction with the procedure)

Revision surgery (including reoperation)

Adverse events (total and specific)

Death

Additional domains for consideration for reporting in TJR clinical trials

Patient participation

Cost

Data are presented as median (IQR) unless otherwise stated.

TJR, total joint replacement.

in a random way for participating and will distribute the link at the annual congress of the 'Deutsche Gesellschaft für Physiotherapiewissenschaft'. In case the needed number of group B participants is not reached with this procedure, the leading physiotherapy societies will be contacted for distributing surveys with a shared link to physiotherapists fitting the inclusion criteria. To include group $\mathrm{C}$ participants, the orthopaedic surgeons society $\mathrm{AE}$ will be contacted for distributing the surveys to their members at their annual congress or per email. Because of the few expected participants fitting the inclusion criteria for surgeons, random selection is not feasible for group C.

\section{Outcomes}

The survey will ask for individual characteristics of the three stakeholder groups, whether the surgeon or physiotherapist is a clinician, researcher or both, and to rate the importance of the items in the core domains set of the OMERACT TJR CDS-G1.0. Participants have to rate every single core domain on a Likert scale (1-9), which was developed by the Grading of Recommendations Assessment, Development and Evaluation working group and used by the OMERACT group for several studies on developing of treatment guidelines and on ranking of core domains. ${ }^{1517} \mathrm{~A}$ rating of $1-3$ will indicate a domain of limited or no importance. A domain rated 4-6 means 
it is important, but still not critical and 7-9 means it is a critically important domain. The overall aim is to determine whether consensus exists within and between the stakeholder groups within the three selected countries.

\section{Sample size}

There is a lack of agreement on how to estimate sample sizes for Delphi studies and endorsements, many published Delphi studies use panels consisting of 10-100 panellists. ${ }^{20}$ There is no reliable data available about the total number of physiotherapists working with patients with TJR or doing research in this field in Germany. About 20000 physiotherapists work in German hospitals, but only a few of them have contact to patients with TJR. Surgery is being done in 1160 hospitals for TKA and 1229 hospitals for THA. ${ }^{21}{ }^{22}$ The estimated sample size for this study will be 100 per stakeholder group. We will be enrolling approximately 50 patients with THA and 50 patients with TKA. We anticipate this will provide similar numbers of men and women. We anticipate a slightly greater proportion of people older than 65 years $(60 \%-$ $65 \%$ ) in our sample, and therefore our subgroup with younger people will be a bit smaller. We believe that even with sample, we will have enough for subgroup analyses.

\section{Study process}

The expert panel will be formed as described. The validation process of the German version will start after publishing this protocol and is estimated to take 6 months. Once the German version of the survey is validated, a phase of 3 months will be used for data collection. Participants will be provided with a link from LimeSurvey, a copy of the survey via email, or receive a paper version of the survey. An accompanying cover letter will ask the participants to complete the survey and send it back once filled in.

On receipt of responses from the different stakeholders, the working group will organise all data in Excel sheets separately for each stakeholder group. The study itself is planned to be finished within 1 year after protocol publication. Following this, data will be statistically analysed and interpreted. In case the data provide reasons for modifications of the core domain set, this will be discussed within the working group, and changes will be implemented, as needed. As to be seen in the flow chart (figure 1), the collected data will be used to achieve further endorsement and consensus among the leaders of orthopaedic international societies and the OMERACT.

\section{Data analysis}

Data analysis will be performed using the statistical software R (V.3.5.1 or newer). The three stakeholder groups will be described according to gender, age category and working background. Descriptive statistics will comprise numbers with corresponding percentages. Statistics will be generated separately for each of the three stakeholder groups, including median (IQR, a measure of variance) ratings for each of the domains within each group as well as median (IQR) ratings for the following patients with TJR subgroups: men versus women, $<55$ years versus $\geq 55$ years and THA versus TKA. Ratings for each domain will be compared between patients versus surgeons versus physiotherapists, as well as between the different subgroups of patients, using a Kruskal-Wallis test when comparing three groups and a Mann-Whitney test when comparing two groups. A p value $<0.05$ will be considered significant. Data will be presented in tables 2-4. Templates for the tables are attached to this protocol.

\section{Patient and public involvement}

OMERACT has its own patient research partner network to ensure patient's voices in core outcome set development. Patients were involved in the development of this OMERACT TJR core domain set from the very beginning and they will be stakeholders in the translation process and in the endorsement described in this protocol.

\section{DISCUSSION}

The inclusion of different stakeholder groups in this survey offers unique opportunities and challenges. We may reach consensus about the TJR core domain set that is concordant with the consensus achieved in the USA and Australia.

We anticipate several study limitations. The sample size may not be large for all subgroup analyses, since we balanced feasibility with desired size of the study sample. We anticipate enough people in the subgroups. It is possible that new suggestions may be noted for inclusion in the core domain set, despite a rigorous process we previously followed with multistakeholder input and multiple rounds of validation and the use of independent samples. We have prespecified the thresholds for inclusion of new domains in the core domain set during cross-cultural validation. We recognise the validation in a German population does not represent the cultural diversity of the whole European population, and we consider this an initial step, anticipating that researchers in other countries will also test these in their populations.

Our study has several strengths as well. With crosscultural validation of this core domain set, we will ensure its generalisability across language and culture. Since we are following the same rigorous methodology as we previously followed, results can be directly replicated and compared with the previous studies, and variations noted.

We expect to get insights into the intergroup and intragroup homogeneity or heterogeneity among physiotherapists, surgeons and patients. It can be hypothesised that healthcare stakeholders such as physiotherapists or surgeons may rate different domains more important than patient stakeholders. For sustainable and generalisable research, consensus about core domains between different stakeholders, are of upmost importance. Deepening knowledge about consensus in those groups, respecting multicultural diversity, will help to harmonise clinical trials on TJR. When finally, research on the same 
topic reports the same outcomes, first the comparability and then the level of evidence for healthcare interventions will increase.

\section{Trial status}

When this manuscript was submitted, recruitment had not yet begun.

\section{Ethics and dissemination}

This study involves human participants. All authors declare that international ethical standards are consistently adhered. Ethical approval has been obtained by the Brandenburg Technical University Research Ethics Committee (BTU-CS, EK 2019-2). The current protocol version number is 2.5 from the 6 May 2020. The consent for participation will be asked for in LimeSurvey, before starting to answer the survey. This manuscript does not contain any individual person's data apart from the authors'. All authors agreed on publishing their contact details. We will publish the results in peer-reviewed publications and at international conferences.

\section{Author affiliations}

${ }^{1}$ Therapy Sciences, Brandenburg University of Technology Cottbus-Senftenberg, Senftenberg, Germany

${ }^{2}$ Division of Clinical Immunology and Rheumatology, University of Alabama at Birmingham, Birmingham, Alabama, USA

${ }^{3}$ Medicine Service, Birmingham Veterans Affairs Medical Center, Birmingham, Alabama, USA

${ }^{4}$ University Hospital of Orthopedics and Trauma Surgery Pius-Hospital, Medical Campus University Oldenburg, Oldenburg, Germany

${ }^{5}$ Musculoskeletal Statistics Unit, The Parker Institute, Frederiksberg, Denmark ${ }^{6}$ Integrative Rheumatology and Orthopedics Center of Excellence, Weill Cornell Medicine, Hospital for Special Surgery, New York, New York, USA

${ }^{7}$ Department of Surgery, St. Vincent's Hospital Melbourne, The University of Melbourne, Melbourne, Victoria, Australia

${ }^{8}$ Klinik für Orthopädie, Sportmedizin und Rehabilitation, Krankenhaus MarkischOderland GmbH, Wriezen, Germany

Contributors RP wrote the manuscript. He will, together with $\mathrm{HH}$, coordinate the project in Germany and will recruit, together with CK, physiotherapists for the validation of the German survey and for the endorsement. JAS, SG and PC will coordinate the project worldwide and make the major methodical decisions. JAS, SG, SM, GHS, PC, SMN and CK reviewed the content of each manuscript draft critically and made substantial contributions to the writing and content. RS is writing the registration in the German trials registry and will lead contributions to the method section. SMN and JAS are responsible for data analyses of the whole project and especially for this manuscript. SG and GHS reviewed the language and writing style. SG makes substantial contributions to the consensus process. HH will recruit patients and orthopaedic surgeons for the validation of the German survey and for the endorsement and will coordinate the inclusion and data collection of those stakeholders. He is responsible for acquisition of necessary funding. All aforementioned authors are part of the expert team for discussion regarding the back translated English version of the survey to identify problems within the translation and validation process. Furthermore, all aforementioned authors will review the final version of the manuscript critically.

Funding The Parker Institute, Bispebjerg and Frederiksberg Hospital is supported by a core grant from the 0ak Foundation (grant number OCAY-13-309). The Oak Foundation had no role in the study design, writing the protocol, or the decision to submit the final protocol for publication. The used funding for preparation and publishing of this project is institutional funding of the Brandenburg University of Technology Cottbus - Senftenberg and the Klinikum Märkisch Oderland GmbH. No external and especially no commercial funding has been used or will be used for this project.

Competing interests RP, GHS, SMN, SG, SM, CK, RS and HH do not have competing interests. JAS has received consultant fees from Crealta/Horizon,
Medisys, Fidia, UBM LLC, Medscape, WebMD, Clinical Care options, Clearview healthcare partners, Putnam associates, Spherix, the National Institutes of Health and the American College of Rheumatology. JAS owns stock options in Amarin pharmaceuticals and Viking therapeutics. JAS is a member of the executive of OMERACT, an organisation that develops outcome measures in rheumatology and receives arms-length funding from 36 companies. JAS serves on the FDA Arthritis Advisory Committee. JAS is a member of the Veterans Affairs Rheumatology Field Advisory Committee. JAS is the editor and the Director of the UAB Cochrane Musculoskeletal Group Satellite Center on Network Meta-analysis. JAS previously served as a member of the following committees: member, the American College of Rheumatology's (ACR) Annual Meeting Planning Committee (AMPC) and Quality of Care Committees, the Chair of the ACR Meet-the-Professor, Workshop and Study Group Subcommittee and the cochair of the ACR Criteria and Response Criteria subcommittee. PC received institutional support from Medacta. PC also received consultancies from Johnson \& Johnson and Stryker. PC received royalties from Johnson and Johnson as part of an instrument design team. PC is part of a Federal Government (Australian) funded industry led consortium (Innovative Manufacturing Cooperative Research Centre) investigating advanced manufacturing in prosthetic design and manufacture. PC is a recipient of National Health and Medical Research Council grants, as well as Australian Research Council grants.

Patient and public involvement Patients and/or the public were involved in the design, or conduct, or reporting, or dissemination plans of this research. Refer to the Methods section for further details.

Patient consent for publication Not required.

Provenance and peer review Not commissioned; externally peer reviewed.

Open access This is an open access article distributed in accordance with the Creative Commons Attribution Non Commercial (CC BY-NC 4.0) license, which permits others to distribute, remix, adapt, build upon this work non-commercially, and license their derivative works on different terms, provided the original work is properly cited, appropriate credit is given, any changes made indicated, and the use is non-commercial. See: http://creativecommons.org/licenses/by-nc/4.0/.

ORCID iDs

Robert Prill http://orcid.org/0000-0002-4916-1206

Jasvinder A Singh http://orcid.org/0000-0003-3485-0006

Robert Schulz http://orcid.org/0000-0002-4830-309X

\section{REFERENCES}

1 Elders MJ. The increasing impact of arthritis on public health. $J$ Rheumatol Suppl 2000;60:6-8.

2 Hunter DJ, Felson DT, Osteoarthritis FDT. Osteoarthritis. BMJ 2006;332:639-42.

3 Aresti N, Kassam J, Nicholas N, et al. Hip osteoarthritis. BMJ 2016;354:i3405.

4 Evans JT, Walker RW, Evans JP, et al. How long does a knee replacement last? A systematic review and meta-analysis of case series and national registry reports with more than 15 years of followup. Lancet 2019;393:655-63.

5 Grimberg A, Janson V, Liebs T, et al. Jahresbericht 2016. Deutschland: Endoprothesenregister, 2016.

6 American joint replacement registry. annual report 2017;4.

7 Australian Orthopaedic Association National Joint Replacement Registry (AOANJRR). Hip, Knee \& Shoulder Arthroplasty: 2018 Annual Report. Adelaide: AOA 2018.

8 Riddle DL, Stratford PW, Bowman DH. Findings of extensive variation in the types of outcome measures used in hip and knee replacement clinical trials: a systematic review. Arthritis Rheum 2008;59:876-83.

9 Riddle DL, Stratford PW, Singh JA, et al. Variation in outcome measures in hip and knee arthroplasty clinical trials: a proposed approach to achieving consensus. J Rheumatol 2009;36:2050-6.

10 Wall PDH, Richards BL, Sprowson A, et al. Do outcomes reported in randomised controlled trials of joint replacement surgery fulfil the OMERACT 2.0 filter? A review of the 2008 and 2013 literature. Syst Rev 2017;6:106.

11 Richards BL, Wall PDH, Sprowson AP, et al. Outcome measures used in arthroplasty trials: systematic review of the 2008 and 2013 literature. J Rheumatol 2017;44:1277-87.

12 Singh JA, Dohm M, Sprowson AP, et al. Outcome domains and measures in total joint replacement clinical trials: can we harmonize them? an OMERACT collaborative initiative. J Rheumatol 2015;42:2496-502. 
13 Singh JA, Dowsey MM, Dohm M, et al. Achieving consensus on total joint replacement trial outcome reporting using the OMERACT filter: endorsement of the final core domain set for total hip and total knee replacement trials for endstage arthritis. J Rheumatol 2017:44:1723-6.

14 Singh JA, Dowsey M, Choong PF. Patient endorsement of the outcome measures in rheumatology (OMERACT) total joint replacement (TJR) clinical trial draft core domain set. BMC Musculoskelet Disord 2017;18:111.

15 Singh JA, Dohm M, Choong PF. Consensus on draft OMERACT core domains for clinical trials of total joint replacement outcome by orthopaedic surgeons: a report from the International consensus on outcome measures in TJR trials (I-COMiTT) group. BMC Musculoskelet Disord 2017;18:45.

16 Hoang A, Goodman SM, Navarro-Millán IY, et al. Patients and surgeons provide endorsement of core domains for total joint replacement clinical trials. Arthritis Res Ther 2017;19:267.

17 Singh JA, Dohm M. Towards consensus on the reporting of core outcome domains in total joint replacement clinical trials: the derivation of the preliminary core outcome domain set. $J$ Arthritis 2016;05:6.
18 Imada A, Nelms N, Halsey D, et al. Physical therapists collect different outcome measures after total joint arthroplasty as compared to most orthopaedic surgeons: a new England study. Arthroplast Today 2018;4:113-7.

19 World health Organisation. Process of translation and adaptation of instruments. Available: http://www.who.int/substance_abuse/ research tools/translation/en/ [Accessed 21 Feb 2019].

20 Akins RB, Tolson H, Cole BR. Stability of response characteristics of a Delphi panel: application of bootstrap data expansion. BMC Med Res Methodol 2005;5:37.

21 AQUA-Institut. Bundesauswertung zum Erfassungsjahr 2014: 17/2 - Hüft-Endoprothesen-Erstimplantation: Qualitätsindikatoren. Göttingen: aqua Institut für angewandte Qualitätsförderung und Forschung Im Gesundheitswesen 2015.

22 AQUA-Institut. Bundesauswertung zum Erfassungsjahr 2014: 17/5 - Knie-Totalendoprothesen-Erstimplantation: Qualitätsindikatoren. Göttingen: aqua Institut für angewandte Qualitätsförderung und Forschung Im Gesundheitswesen GmbH 2015. 